\title{
Qualidade de vida e eventos adversos de pacientes com epilepsia farmacorresistente em uso de lamotrigina
}

\author{
Quality of life and adverse events of patients with drug-resistant epilepsy using lamotrigine
}

André de Oliveira Baldoni ${ }^{1}$, Priscila Freitas-Lima², Veriano Alexandre ${ }^{3}$, Karolini de Faria Mota ${ }^{4}$,

Edson Zangiacomi Martinez ${ }^{5}$, Americo Ceiki Sakamoto5${ }^{5}$, Leonardo Regis Leira Pereira ${ }^{6}$

\begin{abstract}
RESUMO
Modelo do estudo: Estudo transversal. Introdução: O tratamento farmacológico é a primeira opção para o tratamento da epilepsia, e cerca de $40 \%$ dos pacientes necessitam de politerapia para melhor controle das crises epilépticas, o que pode estar associado ao aumento de eventos adversos e comprometimento da qualidade de vida. Objetivos: Avaliar a qualidade de vida de pacientes com epilepsia farmacoresistente em uso de lamotrigina (LTG), bem como verificar a associação dos eventos adversos dos antiepilépticos com a qualidade de vida. Metodologia: Este estudo transversal foi realizado com 75 pacientes com epilepsia farmacorresistente em uso LTG atendidos em um Ambulatório de Epilepsia de Difícil Controle de Ribeirão Preto-SP, no período de maio/2011 a abril/2012. As variáveis clínicas analisadas foram a qualidade de vida (Quality of Life in Epilepsy - Qolie-31) e o perfil de eventos adversos (AEP - Adverse Events Profile Questionnaire). Dados sociodemográficos e farmacoterapêuticos foram coletados através dos prontuários dos pacientes. Este trabalho foi aprovado pelo Comitê de Ética em Pesquisa do Hospital das Clínicas da Faculdade de Medicina de Ribeirão Preto da Universidade de São Paulo (HCFMRP-USP), cujo número do processo é 8791/2010. Resultados: Os eventos adversos mais frequentes foram sonolência e dificuldade de concentração. Além disso, observou-se baixos escores em todos os domínios relacionados à qualidade de vida (Qolie-31). Conclusão: A qualidade de vida possui associação inversamente significativa com os eventos adversos, nos pacientes em uso de antiepilépticos $(p<0,01)$.
\end{abstract}

Palavras-chave: Lamotrigina. Epilepsia Resistente a Medicamentos. Qualidade de Vida. Efeitos Colaterais e Reações Adversas Relacionados a Medicamentos.

\section{ABSTRACT}

Study design: Cross-sectional study. Introduction: Pharmacological treatment is the first option to treat epilepsy, and about $40 \%$ of patients require polytherapy to better control epileptic seizures, which may be associated with an increase in adverse events and impairment of quality of life.

1. Docente, Universidade Federal de São João Del-Rei (UFSJ), campus Divinópolis-MG.

2. Docente do Centro Universitário Barão de Mauá e da Faculdade de Taquaritinga (FTGA).

3. Doutor pela Faculdade de Medicina de Ribeirão Preto, Universidade de São Paulo (FMRP-USP).

4. Farmacêutica, mestre pela Universidade Federal de São João Del-Rei (UFSJ).

5. Docente, Faculdade de Medicina de Ribeirão Preto, (FMRP-USP).

6. Docente, Faculdade de Ciências Farmacêuticas de Ribeirão Preto, (FCFRP-USP).
CORRESPONDÊNCIA: André de Oliveira Baldoni. Universidade Federal de São João del-Rei (UFSJ) Campus Centro-Oeste Dona Lindu (CCO) Rua Sebastião Gonçalves Coelho, 400, Bairro Chanadour, CEP 35.501-296, Divinópolis - MG, Brazil.

Recebido em 30/01/2018 Aprovado em 31/08/2018 
Objective: To evaluate the quality of life of patients with pharmacoresistant epilepsy using lamotrigine (LTG), as well as to verify the association of adverse events with antiepileptic drugs with quality of life. Methods: This cross-sectional study was carried out with 75 patients with drugresistant epilepsy using LTG, in a Clinic of Epilepsy of Difficult Control of Ribeirão Preto-SP, from May/2011 to April / 2012. The clinical variables analyzed were Quality of Life in Epilepsy (QOLIE31) and the Adverse Events Profile Questionnaire (AEP). Socio-demographic and pharmacotherapeutic data were collected through patient records. This study was approved by the Research Ethics Committee of the General Hospital of the Medical School of Ribeirão Preto of the University of São Paulo (HCFMRP-USP), whose case number is 8791/2010. Results: The most frequent adverse events were somnolence and difficulty concentrating. In addition, low scores were observed in all domains related to quality of life (Qolie-31). Conclusion: Quality of life has an inverse association with adverse events in patients using antiepileptic drugs $(p<0.01)$.

Keywords: Lamotrigine. Drug Resistant Epilepsy. Quality of Life. Adverse Drug Events.

\section{INTRODUÇÃO}

A epilepsia é uma desordem neurológica crônica caracterizada pela presença de crises epilépticas recorrentes, gerando consequências terapêuticas, cognitivas, psicológicas e sociais aos acometidos ${ }^{1}$. Embora nas últimas décadas a clínica tenha experimentado avanços nas opções para seu tratamento, o medicamento continua sendo a primeira escolha ${ }^{2}$. No entanto, até $40 \%$ dos pacientes não respondem satisfatoriamente ao tratamento farmacológico e são considerados farmacorresistentes, portanto torna-se necessária a associação de dois ou mais fármacos antiepilépticos (FAE) para alcançar o controle das crises, sendo a LTG, um FAE de segunda geração, amplamente utilizado nesta situação clínica ${ }^{3}$. Apesar do benefício para o controle das crises, a politerapia favorece o aparecimento de interações medicamentosas e eventos adversos ${ }^{4}$.

O impacto dos FAE de segunda geração no tratamento das crises epilépticas e na qualidade de vida dos indivíduos com epilepsia farmacorresistente ainda não foi completamente esclarecido, após sua introdução no mercado mundial. Uma metanálise mostrou que apenas um em cada cinco pacientes em uso de FAE modernos, apresenta uma redução de $50 \%$ das crises epiléticas ${ }^{5}$. Ensaios clínicos randomizados mostraram que, entre 10 e $15 \%$ dos pacientes com epilepsia farmacorresistente alcançaram a remissão total das crises utilizando FAE de segunda geração, e mesmo assim, esses períodos de remissão foram considerados curtos. Vale ressaltar que a utilização de FAE em estudos clínicos é diferente da sua prescrição na prática clínica diária6 .

Adicionalmente, estudos observacionais têm demonstrado que a redução na frequência das crises epilépticas, quando comparada ao controle total destas por um longo período, apresenta pouco impacto sobre a qualidade de vida dos pacientes ${ }^{7}$. Diante disso, o presente estudo tem por objetivo avaliar a qualidade de vida de pacientes com epilepsia farmacorresistente em uso de LTG, bem como verificar a associação dos eventos adversos dos antiepilépticos com a qualidade de vida.

\section{CASUÍSTICA E MÉTODOS}

\section{Delineamento, local e população do estudo}

Este estudo transversal foi realizado de maio de 2011 a abril de 2012 no Ambulatório de Epilepsia de Difícil Controle (AEDC) do Hospital das Clínicas da Faculdade de Medicina de Ribeirão Preto da Universidade de São Paulo (HC-FMRP-USP).

Foram convidados todos os pacientes acompanhados pelo AEDC e que atendessem aos seguintes critérios de inclusão: (i) idade igual ou superior a 18 anos; (ii) diagnóstico de epilepsia farmacorresistente; e (iii) em uso de LTG, em mono ou politerapia, pelo menos por dez dias em dose estável. Os critérios de exclusão utilizados foram: (i) gestantes; (ii) pacientes com evidências de doença hepática e renal.

Os pacientes foram identificados pelo sistema de prescrição eletrônica do hospital e, posteriormente, os que atenderam aos critérios de 
inclusão foram contatados para realização da entrevista no dia da consulta médica previamente agendada.

\section{Variáveis do estudo}

As variáveis clínicas obtidas por meio de fontes de informações primárias (questionários validados) foram:

- Eventos adversos: utilizou-se o Adverse Events Profile Questionnaire $(A E P)^{8}$. Este questionário possui 19 itens que correspondem a diferentes eventos adversos. Cada um desses eventos apresenta uma escala que varia de um a quatro pontos de acordo com a frequência de ocorrência ( 1 = nunca; 2 = raramente; $3=$ algumas vezes; 4 = sempre ou frequentemente) (GI LLIAM et al., 2004). O escore total varia de 19 a 76 pontos, sendo que valores maiores refletem maior frequência de eventos adversos.

- Qualidade de vida: variável coletada segundo o questionário Quality of Life in Epilepsy-31 (Qolie31), que é um instrumento específico estruturado para a avaliação da qualidade de vida em pacientes com epilepsia9. O questionário inclui um item sobre o estado de saúde geral e 30 itens subdivididos em sete domínios: preocupação com as crises, bem-estar emocional, energia/fadiga, funcionamento social, funcionamento cognitivo, eventos adversos dos medicamentos e qualidade de vida em geral. O paciente deve respondê-lo com base na percepção da sua qualidade de vida nas últimas quatro semanas. O escore total varia de zero a 100 pontos, sendo que os valores maiores refletem melhor qualidade de vida.

Foram utilizadas fontes secundárias (registros nos prontuários dos pacientes) para obtenção das demais informações clínicas. Dessa forma, foram coletas as seguintes variáveis: diagnóstico da epilepsia contendo tipo de crise e síndrome epiléptica; comorbidades médicas e eventos adversos.

Além destas, as variáveis farmacoepidemiológicas coletadas foram:

- Tratamento farmacológico prescrito (medicamentos e posologia): esta informação foi obtida junto ao prontuário do paciente e confirmada durante a entrevista;
- Presença de fármacos indutores e inibidores do metabolismo da LTG. Para a análise descritiva e analítica, os medicamentos utilizados pelos entrevistados foram categorizados em quatro grupos:

1. Monoterapia: pacientes que utilizam apenas LTG ou FAE que não interferem na concentração plasmática da LTG;

2. Indutores: pacientes em uso de LTG juntamente com fármacos indutores enzimáticos;

3. Inibidores: pacientes em uso de LTG juntamente com fármacos inibidores enzimáticos;

4. Indutores + Inibidores: pacientes em uso de indutores e inibidores enzimáticos concomitantemente.

\section{Análise estatística}

As variáveis foram categorizadas, sendo que para as variáveis contínuas utilizou-se a mediana dos valores obtidos como ponto de corte, exceto para variáveis que apresentam valores com alguma representação na prática clínica, como o AEP, em que valores iguais ou superiores a 45 pontos são indicadores de toxicidade, necessitando de possível intervenção do clínico $^{10}$.

O teste $\mathrm{t}$ de Student foi utilizado para analisar possíveis diferenças entre: o registro de evento adverso no prontuário médico com o escore AEP e os escores do Qolie-31. Para analisar a associação entre os escores AEP e os escores do Qolie-31 realizou-se o teste de Correlação de Pearson.

As análises estatísticas foram realizadas utilizando o software SAS (Statistical Analysis System), versão 9.2, e o software $R$, versão 2.15.3. Para testar as hipóteses o nível de significância foi fixado em á =0,05, e intervalo de confiança de $95 \%$.

\section{Aspectos Éticos}

O trabalho foi aprovado pelo Comitê de Ética em Pesquisa do HCFMRP-USP, cujo número do processo é $8791 / 2010$.

\section{RESULTADOS E DISCUSSÃO}

Dos 75 pacientes adultos incluídos neste estudo, houve predomínio da faixa etária de 1860 anos $(97 \%)$, assim como um predomínio do 
sexo masculino (53\%). Em média, o peso corporal dos pacientes foi de $74 \mathrm{~kg}$, variando de 48 a $145 \mathrm{~kg}$.

\section{Perfil Farmacoepidemiológico}

Em relação ao perfil farmacoepidemiológico dos pacientes incluídos no estudo observou-se que os pacientes utilizam de um a quatro $F A E$, sendo a média de 2,8 $\mathrm{FAE} /$ paciente. A politerapia (uso de dois ou mais FAE) foi identificada em $97 \%$ dos casos, sendo que $61 \%$ dos pacientes utilizavam três fármacos, $24 \%$ utilizavam dois e $12 \%$ utilizavam quatro FAE para o tratamento da epilepsia farmacorresistente. Esta elevada prevalência de politerapia (97\%) identificada no presente estudo pode estar associada à dificuldade e complexidade do regime terapêutico utilizado para tratar os pacientes com epilepsia farmacorresistente.

Considerando tal prevalência de politerapia, é importante mencionar que ensaios clínicos randomizados não evidenciam que esquemas de politerapia sejam mais efetivos do que monoterapias para controle das crises epilépticas ${ }^{11}$. No entanto, a escolha entre monoterapia ou politerapia é uma decisão que dependerá da resposta clínica individualizada e da anamnese clínica realizada junto a cada paciente.
Analisando os esquemas de politerapia, identificou-se que a carbamazepina (CBZ) e o clobazam (CLB) foram os fármacos com maior prevalência de uso associado à LTG, $67 \%$ e $65 \%$, respectivamente. Esse resultado corrobora com a elevada frequência de pacientes com epilepsia do tipo focal, que é a indicação principal da CBZ e da LTG (Figura 1) ${ }^{12}$.

Apesar da associação de LTG com CBZ ser comum na prática clínica, tanto para adultos quanto para crianças, é importante ressaltar que esses dois FAE apresentam semelhanças farmacodinâmicas, e por este motivo a racionalidade desta associação é questionada por alguns estudos ${ }^{13}$. Além disso esta associação pode aumentar as chances de eventos adversos relacionados a neurotoxicidade tais como sonolência, diplopia e ataxia ${ }^{14}$. Entretanto, frente à dificuldade no controle das crises dos pacientes com epilepsia farmacorresistente e diante da ausência de outras opções terapêuticas disponíveis no Brasil na época de condução do presente estudo, esta associação torna-se, frequentemente, necessária.

Tais razões previamente discutidas podem repercutir, também, sobre a elevada prevalência de uso do CLB (65\%), visto que, quando comparada a outros países, a frequência de uso deste fármaco em pacientes com epilepsia é cerca de 4,5 ve-

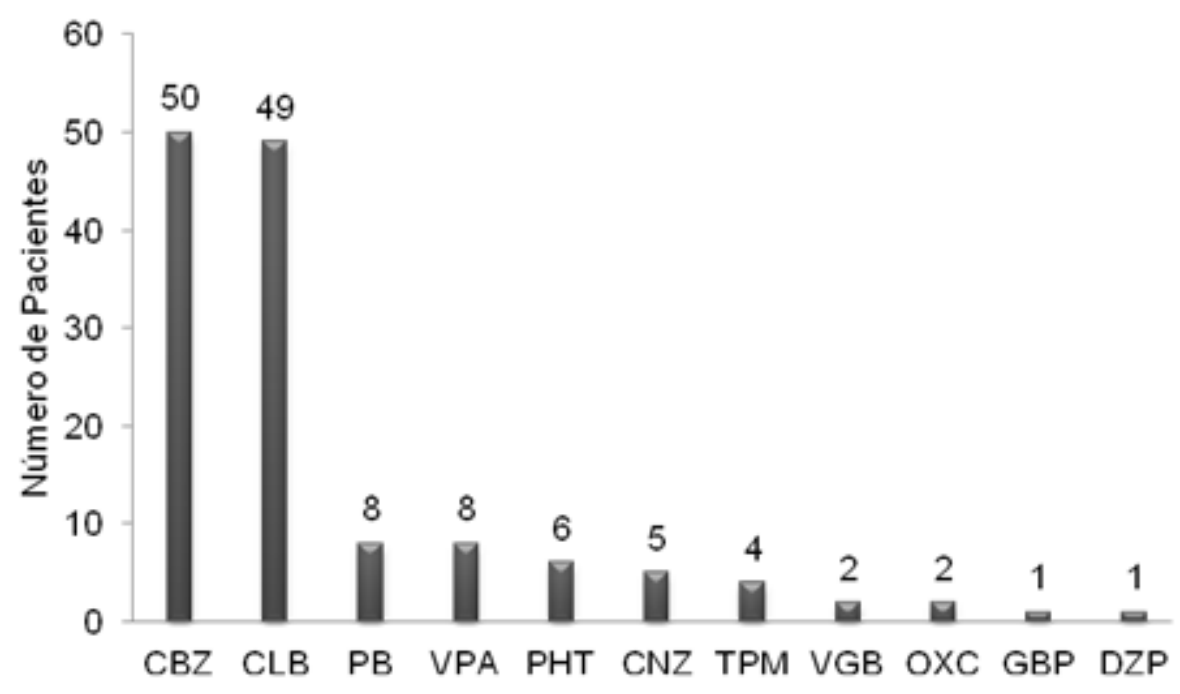

Figura 1. Fármacos antiepilépticos utilizados concomitantemente com a lamotrigina pelos pacientes adultos com epilepsia refratária atendidos no AEDC do HCFMRP-USP no período de maio/2011 a abril/2012 ( $n=75)$ (CBZ: carbamazepina; CLB: clobazam; PB: fenobarbital; VPA: valproato; PHT: fenitoína; CNZ: clonazepam; TPM: topiramato; VGB: vigabatrina; OXC: oxcarbazepina; GBP: gabapentina; DZP: diazepam). 
zes maior. Um estudo realizado na Noruega identificou que os benzodiazepínicos foram prescritos para apenas $14 \%$ dos pacientes com epilepsia ${ }^{15}$. Outro estudo realizado na Itália observou que a prevalência de uso de CLB em pacientes adultos com epilepsia farmacorresistentes é de $18 \%^{16}$. Neste contexto é relevante destacar que o CLB foi recentemente aprovado nos Estados Unidos para ser utilizado como adjuvante de crises associadas à síndrome de Lennox-Gastaut ${ }^{17}$. Todavia, este fármaco, há décadas, tem se mostrado efetivo como farmacoterapia adjuvante no tratamento dos diversos tipos de epilepsia farmacorresistente, especialmente em casos de crises parciais ${ }^{18}$.

A baixa prevalência de uso dos demais FAE pode estar associada à indicação de uso, custos, perfil farmacocinético, frequência e gravidade de eventos adversos. Por exemplo, a PHT, apesar de apresentar baixo custo e ser efetiva em casos de crises parciais, possui um perfil farmacocinético que dificulta o ajuste posológico na prática clínica, e, além disso, possui um amplo perfil de interações medicamentosas, inclusive com contraceptivos orais a base de estrogênio, além dos eventos adversos cognitivos e cosméticos (hirsutismo e hiperplasia gengival) que podem limitar o seu uso, principalmente em mulheres na idade fértil ${ }^{19}$.

Especificamente no que diz respeito à baixa prevalência de uso dos outros FAE de segunda geração (VGB, TPM e OXC), é relevante destacar que todos os FAE de segunda geração são indicados como terapia adjuntiva em pacientes adultos com epilepsia parcial farmacorresistentes ${ }^{20}$. Estudos realizados nos últimos anos demonstram uma tendência mundial de aumento de prescrição dos FAE de segunda geração, visto que eles apresentam menor frequência de reações adversas e um perfil de farmacocinético conveniente ${ }^{15}$.

Em relação às doses de LTG, foi notado que a maioria dos pacientes (52\%) utiliza doses acima da recomendação diária média de manutenção para adultos (Defined Daily Dosage - DDD) que é de $300 \mathrm{mg} / \mathrm{dia}$, sendo $500 \mathrm{mg} / \mathrm{dia}$ a dose com maior prevalência de uso $(21,3 \%)$. Esta prevalência de doses consideradas elevadas também pode estar associada à complexidade do tratamento farmacológico e pela dificuldade no controle das crises, situações clínicas comuns em pacientes com epilepsia farmacorresistente.

\section{Eventos adversos}

Analisando o perfil de eventos adversos dos 73 pacientes que responderam ao AEP, verificouse que as queixas mais prevalentes estavam associadas ao Sistema Nervoso Central (SNC), sendo sonolência, dificuldade de concentração e nervosismo e/ou agitação as mais comumente reportadas. Resultado semelhante foi encontrado por um estudo realizado no Reino Unido, o qual identificou cansaço, problema de memória e dificuldade de concentração como os eventos adversos mais prevalentes quando analisados pacientes com diversos tipos de epilepsia, em uso de monoterapia e politerapia ${ }^{21}$.

A análise quantitativa do AEP identificou que 33 pacientes $(44 \%)$ apresentaram escore total do AEP igual ou superior a 45 pontos. Segundo Gilliam e colaboradores (2004), este valor representa risco de intoxicação e, muitas vezes, exige alguma intervenção clínica (Figura 2).

O perfil de eventos adversos é influenciado por causas multifatoriais, e dentre estes fatores destacam-se as comedicações utilizadas pelos pacientes. O estudo realizado por Andrew e colaboradores (2012) comparou os escores de AEP de pacientes em uso de CBZ, VPA, levetiracetam (LEV) e LTG, e observou que os pacientes em uso de CBZ apresentaram os maiores escores ( $n=46$, média $=44,83)$, seguido do LEV $(n=37, n=41,68)$, LTG ( $n=42$, média $=40,05)$ e VPA ( $n=32$, média $=40,03)^{21}$. Estes resultados corroboram com a elevada frequência de eventos adversos identificados entre os pacientes do presente estudo, visto que a CBZ foi o FAE com maior prevalência de uso associado à LTG.

Além da influência individual dos FAE sobre o perfil de eventos adversos, é importante ressaltar que a quantidade de FAE coprescritos também influencia de forma significativa este parâmetro clínico, visto que os pacientes em uso de politerapia apresentam escores mais elevados no AEP ( média $=45,56$ ) quando comparados a pacientes em uso de monoterapia ( édia $=42,29)^{21}$. Esta associação e a prevalência de pacientes em uso de politerapia ( $97 \%$ ) corroboram com os elevados escores do AEP observados neste estudo.

Em relação à análise dos prontuários para identificação de possíveis eventos adversos, 20 


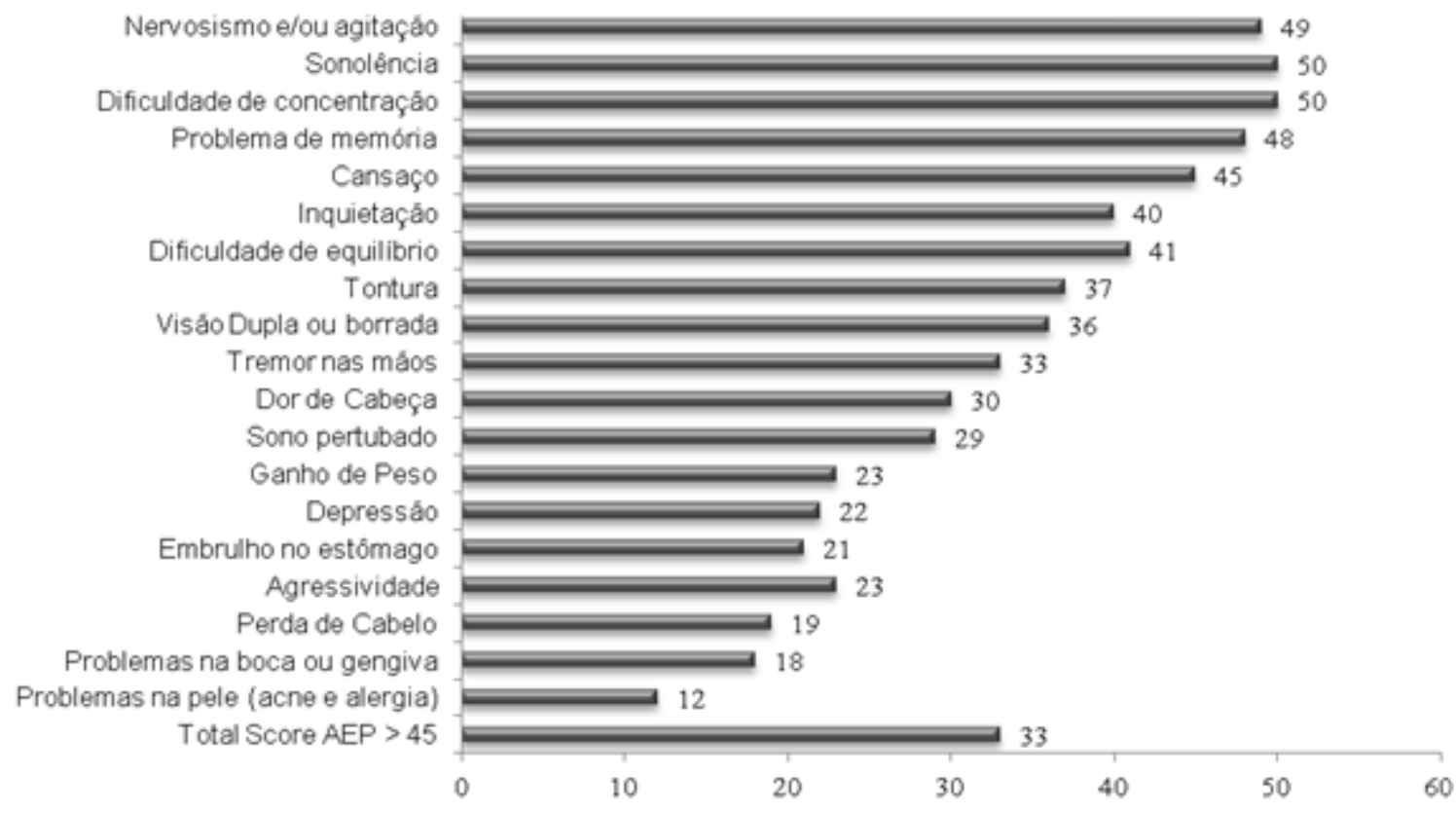

Pacientes que relataram o Evento Adverso ("al gumas vezes" e/ou "frequentemente")

Figura 2. Eventos adversos dos pacientes adultos com epilepsia refratária em uso de lamotrigina atendidos no AEDC do HCFMRP-USP no período de maio/2011 a abril/2012 ( $\mathrm{n}=73$. (AEP: Adverse Events Profile Questionnaire).

prontuários (27\%) dos 75 analisados apresentaram algum registro de evento adverso, sendo os mais prevalentes: sonolência $(n=8)$, diplopia $(n=7)$, tontura $(n=4)$ e acne $(n=3)$. Dessa forma, é possível observar que a sonolência foi o evento adverso mais frequentemente identificado pelos dois métodos utilizados, isto é, aplicação do AEP e análise de prontuários. A análise quantitativa desses eventos, por subgrupos terapêuticos, está apresentada na Tabela 1.
Comparando as duas formas de identificação e quantificação de eventos adversos utilizadas no presente estudo observou-se que, apesar da média dos escores de AEP do grupo que apresentou registro de evento adverso no prontuário ter sido superior $(45,11 \pm 10,79)$ ao grupo sem registro de evento adverso $(41,83 \pm 8,98)$, não foi observada diferença estatisticamente significativa entre estas médias obtidas $(p=0,24)$ (Figura 3 ). Esses resultados sugerem que, neste cen-

Tabela 1

Eventos adversos dos pacientes adultos com epilepsia refratária em uso de lamotrigina atendidos no AEDC do HCFMRP-USP no período de maio/ 2011 a abril/ 2012.

\begin{tabular}{|c|c|c|c|c|}
\hline Variável & Monoterapia & Indutor & Inibidor & Inibidor + Indutor \\
\hline \multicolumn{5}{|l|}{ Evento adverso no prontuário } \\
\hline Presente & 03 & 14 & 01 & 02 \\
\hline Ausente & 02 & 14 & 02 & 00 \\
\hline Informação não disponível & 05 & 29 & 02 & 01 \\
\hline \multicolumn{5}{|l|}{ Escore AEP $(n=73)$} \\
\hline$\geq 45$ pontos & 03 & 26 & 04 & 01 \\
\hline$<45$ pontos & 07 & 29 & 01 & 02 \\
\hline
\end{tabular}

AEP: Adverse Events Profile Questionnaire. 
tro de referência em epilepsia, os clínicos responsáveis pelo atendimento destes pacientes identificam e relatam os eventos adversos durante a anamnese clínica. Além disso, esta constatação confirma a aplicabilidade do AEP na identificação dos eventos adversos na prática clínica e sugere boa acurácia em relação à anamnese clínica.

Em relação ao perfil dos eventos adversos da LTG, a literatura descreve que as queixas mais prevalentes estão relacionadas ao SNC e às reações dermatológicas. O rash cutâneo ocorre em aproximadamente $10 \%$ dos pacientes em uso de LTG, e a incidência de eventos adversos da LTG é maior nos primeiros meses de tratamento, podendo ocorrer, inclusive, casos de falência hepática e renal ${ }^{22}$. No presente estudo foi observada baixa frequência de eventos cutâneos e ausência de eventos adversos graves. Isso pode ser justifi- cado pelo tipo de delineamento do presente estudo e pelo tempo de tratamento dos pacientes incluídos, visto que os estudos transversais não são adequados para identificação de reações adversas do tipo $B$ (reações imprevisíveis e não associadas à farmacodinâmica), como por exemplo, o rash cutâneo. Este tipo de reação exige a retirada do medicamento e recomendações para se evitar o uso futuro; dessa forma, objetivandose analisar a frequência deste tipo de reação recomenda-se a realização de estudos longitudinais de coorte. Ademais, é importante enfatizar que os pacientes incluídos no presente estudo utilizavam os FAE por no mínimo três meses, que é o período médio entre as consultas agendadas no ambulatório. Isso pode ter inviabilizado a identificação de eventos adversos comuns no início do tratamento farmacológico.

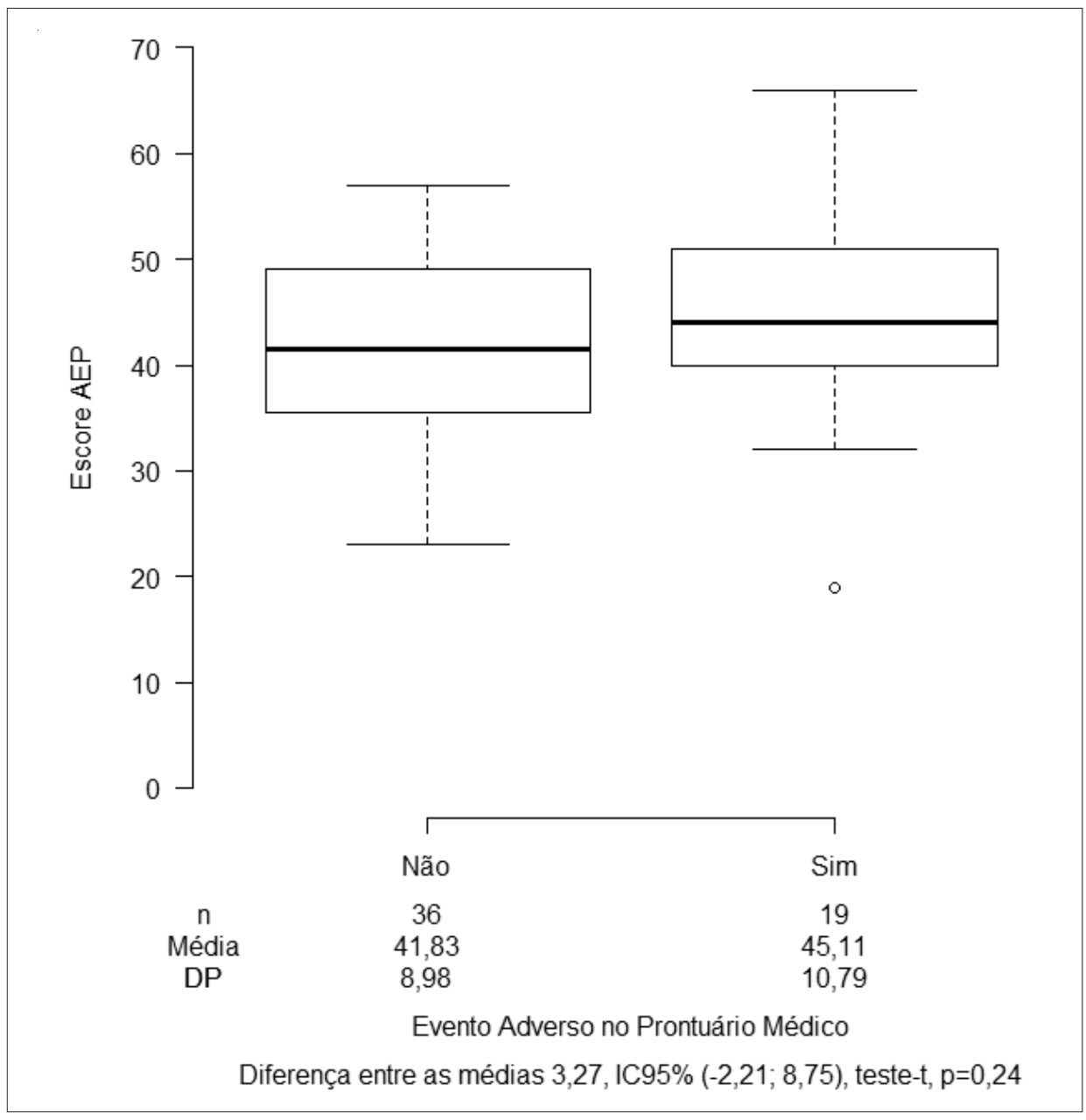

Figura 3. Associação entre escores AEP (Adverse Events Profile) e o registro de evento adverso no prontuário médico dos pacientes adultos com epilepsia refratária em uso de lamotrigina atendidos no AEDC do HCFMRP-USP no período de maio/2011 a abril/2012 ( $\mathrm{n}=75$ ). (DP: Desvio Padrão). 
Sabendo-se destas limitações metodológicas é relevante destacar que o perfil de eventos adversos encontrado no presente estudo não isenta a possibilidade de reações idiossincrásicas graves associadas à LTG e aos demais FAE contidos nos diferentes esquemas terapêuticos analisados. Além disso, durante a análise e a interpretação destes resultados, é preciso considerar a possibilidade de associação desses eventos adversos com os demais FAE, visto que, no presente estudo não foi possível obter amostra representativa de pacientes em uso de LTG em monoterapia.

Neste contexto é pertinente citar os resultados do estudo realizado por Aldenkamp e Baker (2001), os quais identificaram que os efeitos cognitivos apresentados por pacientes em uso de politerapia de FAE não estão associados à LTG, sendo, na maioria das vezes, associados aos FAE de primeira geração ${ }^{23}$. E ainda, os resultados de estudos que compararam grupos de pacientes em uso de monoterapia com LTG, CBZ, PHT e VPA evidenciaram que a LTG apresentou melhores parâmetros relacionados à qualidade de vida e ao perfil de eventos adversos ${ }^{24}$. Em comparação com FAE de segunda geração, Wong e colaboradores (2001) observaram menor incidência de sonolência, irritabilidade, problemas de memória, psicose e cansaço no grupo de pacientes em uso de LTG, quando comparados a pacientes em uso de GBP e VGB.

A diversidade de esquemas terapêuticos identificados no presente estudo inviabilizou uma análise comparativa entre os perfis de eventos adversos (Tabela 2). Para se avaliar e confirmar os eventos adversos que poderiam estar associados à LTG seria ideal e necessário a realização de estudos clínicos com pacientes em uso de LTG em monoterapia, o que não corresponde à realidade dos pacientes com epilepsia farmacorresistente devido à complexidade do plano farmacoterapêutico que esta morbidade exige. Além disso, é importante ressaltar que no Brasil, a LTG é ofertada no Sistema Único de Saúde (SUS) apenas aos pacientes com epilepsia farmacorresistente.

\section{Qualidade de vida}

Os resultados do Qolie-31 apresentaram, em geral, baixos escores em todos os domínios relacionados ao parâmetro humanístico qualida- de de vida, sendo a preocupação com as crises e os eventos adversos dos medicamentos os domínios a que corresponderam maior comprometimento da qualidade vida. Estes resultados são referentes a 62 pacientes, visto que 13 pacientes não conseguiram compreender as questões contidas no instrumento (Tabela 3).

Além dos baixos escores observados nos resultados do Qolie-31, deve-se considerar a elevada prevalência $(17 \%)$ de pacientes que não conseguiram responder às perguntas deste instrumento. Isto pode ser considerado como um indicador de qualidade de vida para estes pacientes, visto que a própria dificuldade de compreensão é um fator que afeta negativamente este parâmetro humanístico, o qual é amplamente influenciado pelas limitações provocadas pela epilepsia de difícil controle.

Esses resultados corroboram com os perfis clínico e farmacoterapêutico da epilepsia farmacorresistente, tendo em vista que este tipo de epilepsia é caracterizada pela elevada frequência de crises epilépticas e um tratamento que exige esquemas terapêuticos complexos, o que, consequentemente, gera eventos adversos que afetam negativamente as atividades cotidianas do paciente. A qualidade de vida dos pacientes entrevistados apresentou associação significativa inversamente proporcional com os eventos adversos obtidos pelo AEP $(r=-0,69, p<0,01)$, sugerindo que, quanto maior os escores do AEP, pior é a qualidade de vida destes pacientes (Figura 4). Esses resultados estão em concordância com estudos prévios realizados por Gilliam e colaboradores (2004) e Alexandre Junior e colaboradores (2011). A evidência desta associação serve de alerta para muitas condutas clínicas atuais, visto que, frequentemente, os parâmetros clínicos e laboratoriais sobrepõem os resultados humanísticos durante a determinação do plano terapêutico.

Analisando a associação entre este parâmetro humanístico e os eventos adversos registrados no prontuário foi observado que, apesar da média dos escores do Qolie-31 ter sido superior em pacientes que não apresentaram registros de eventos adversos no prontuário (média $=45,17$ $\pm 16,94$ ) quando comparado aos que apresentaram $(42,24 \pm 18,29)$, esta análise não apresentou significância estatística ( $p=0,58)$ (Figura 5). 
Tabela 2.

Eventos adversos, de acordo com os diferentes esquemas terapêticos, dos pacientes adultos com epilepsia refratária em uso de lamotrigina atendidos no AEDC do HCFMRP-USP no período de maio/ 2011 a abril/ $2012(n=75)$.

\begin{tabular}{|c|c|c|c|c|c|}
\hline \multirow[b]{2}{*}{ Esquema Terapêutico } & \multicolumn{4}{|c|}{ Escore AEP $(n=73)$} & \multirow{2}{*}{$\frac{\text { Evento adverso no prontuário }(\mathrm{n}=75)}{n}$} \\
\hline & $\mathrm{n}$ & $(\%)$ & Média & $( \pm D P)$ & \\
\hline $\mathrm{LTG}+\mathrm{CBZ}+\mathrm{CLB}$ & 29 & $(39,7)$ & 43 & $(9,50)$ & 06 \\
\hline $\mathrm{LTG}+\mathrm{CBZ}$ & 09 & $(12,3)$ & 45 & $(8,48)$ & 01 \\
\hline LTG + CLB + PHT & 04 & $(5,6)$ & 46 & $(13,23)$ & 01 \\
\hline $\mathrm{LTG}+\mathrm{CBZ}+\mathrm{PB}$ & 03 & $(4,0)$ & 43 & $(6,51)$ & 01 \\
\hline LTG + VPA & 03 & $(4,0)$ & 44 & $(8,66)$ & 00 \\
\hline LTG & 02 & $(2,7)$ & 37 & $(2,12)$ & 01 \\
\hline $\mathrm{LTG}+\mathrm{CNZ}$ & 02 & $(2,7)$ & 48 & $(8,49)$ & 01 \\
\hline $\mathrm{LTG}+\mathrm{CBZ}+\mathrm{CLB}+\mathrm{PB}$ & 02 & $(2,7)$ & 46 & $(0,71)$ & 01 \\
\hline $\mathrm{LTG}+\mathrm{CBZ}+\mathrm{CNZ}$ & 02 & $(2,7)$ & 48 & $(9,19)$ & 01 \\
\hline LTG + CLB & 02 & $(2,7)$ & 37 & $(19,09)$ & 00 \\
\hline $\mathrm{LTG}+\mathrm{CLB}+\mathrm{OXC}$ & 02 & $(2,7)$ & 45 & $(9,90)$ & 00 \\
\hline LTG + CBZ + VPA & 01 & $(1,4)$ & $40 *$ & (NA) & 01 \\
\hline $\mathrm{LTG}+\mathrm{CBZ}+\mathrm{CLB}+\mathrm{VPA}$ & 01 & $(1,4)$ & $66 *$ & (NA) & 01 \\
\hline $\mathrm{LTG}+\mathrm{CLB}+\mathrm{PHT}+\mathrm{VGB}$ & 01 & $(1,4)$ & $49 *$ & (NA) & 01 \\
\hline LTG + CLB + TPM & 01 & $(1,4)$ & $32 *$ & (NA) & 01 \\
\hline LTG + CLB + VPA & 01 & $(1,4)$ & $49 *$ & (NA) & 01 \\
\hline LTG + PB & 01 & $(1,4)$ & $44 *$ & (NA) & 01 \\
\hline $\mathrm{LTG}+\mathrm{CLB}+\mathrm{GBP}+\mathrm{VPA}$ & 01 & $(1,4)$ & $53 *$ & (NA) & 00 \\
\hline $\mathrm{LTG}+\mathrm{CLB}+\mathrm{PB}$ & 01 & $(1,4)$ & $46 *$ & (NA) & 00 \\
\hline LTG + CLB + DZP & 01 & $(1,4)$ & $44 *$ & (NA) & 00 \\
\hline $\mathrm{LTG}+\mathrm{CBZ}+\mathrm{CLB}+\mathrm{TPM}$ & 01 & $(1,4)$ & $42 *$ & (NA) & 00 \\
\hline LTG +TPM & 01 & $(1,4)$ & $40 *$ & (NA) & 00 \\
\hline LTG + CLB + PHT+TPM & 01 & $(1,4)$ & $30 *$ & (NA) & 00 \\
\hline $\mathrm{LTG}+\mathrm{CBZ}+\mathrm{PB}+\mathrm{VPA}$ & 01 & $(1,4)$ & $26 *$ & (NA) & 00 \\
\hline $\mathrm{LTG}+\mathrm{CBZ}+\mathrm{CLB}$ & NA & NA & 01 & & \\
\hline $\mathrm{LTG}+\mathrm{CBZ}+\mathrm{VGB}$ & NA & NA & 00 & & \\
\hline
\end{tabular}

CBZ: carbamazepina; CLB: clobazam; CNZ: clonazepam; DZP: diazepam; GBP: gabapentina; LTG: lamotrigina; OXC: oxcarbazepina; PB: fenobarbital; PHT: fenitoína; TPM: topiramato; VGB: vigabatrina; VPA: valproato. Refere-se ao número absoluto, devido a impossibilidade de cálculo da média; NA: Não se aplica. 
Tabela 3

Qualidade de vida dos pacientes adultos com epilepsia refratária em uso de lamotrigina atendidos no AEDC do HCFMRP-USP no período de maio/ 2011 a abril/ 2012 (n=62).

\begin{tabular}{llc}
\hline Domínio do Qolie- 31 & Média (DP) & Mínimo-Máximo \\
\hline Preocupação com as crises & $37(28,26)$ & $00-100$ \\
Escore total & $43(16,24)$ & $14-83$ \\
Eventos adversos & $46(28,38)$ & $00-100$ \\
Função social & $47(27,45)$ & $00-100$ \\
Função cognitive & $49(27,07)$ & $03-100$ \\
Vitalidade (Energia) & $51(24,59)$ & $00-95$ \\
Bem-estar emocional & $55(22,34)$ & $04-100$ \\
Qualidade de vida geral & $63(18,58)$ & $00-100$
\end{tabular}

Qolie-31: Quality of Life in Epilepsy-31; DP: Desvio-padrão.

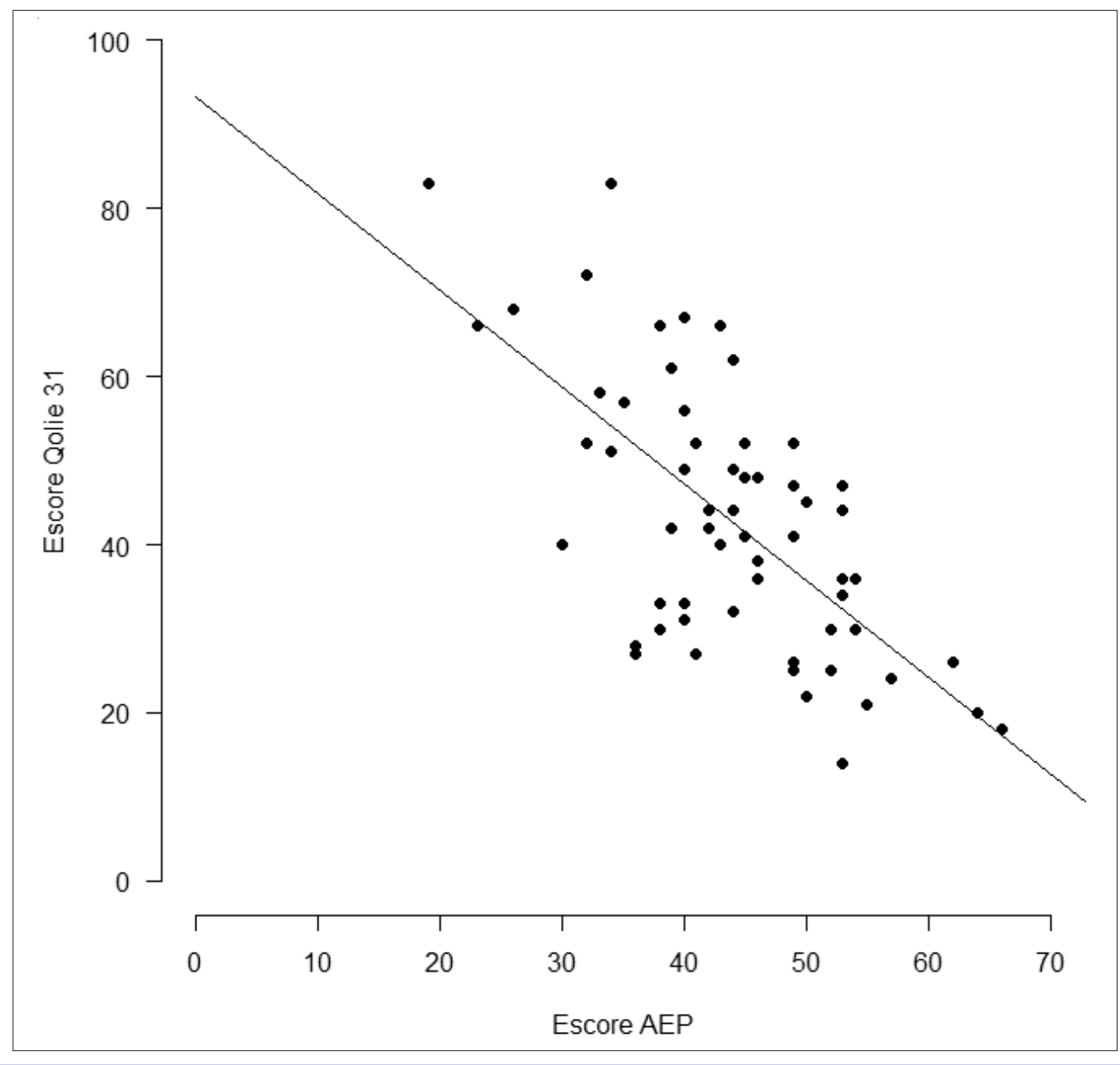

Figura 4. Associação entre os escores do Adverse Events Profile (AEP) e a qualidade de vida (Qolie-31) dos pacientes adultos com epilepsia refratária em uso de lamotrigina atendidos no AEDC do HCFMRP-USP no período de maio/2011 a abril/2012. Correlação de Pearson, $r=-0,69,95 \% \mathrm{Cl}(-0,79,-0,52), p<0,01$. 


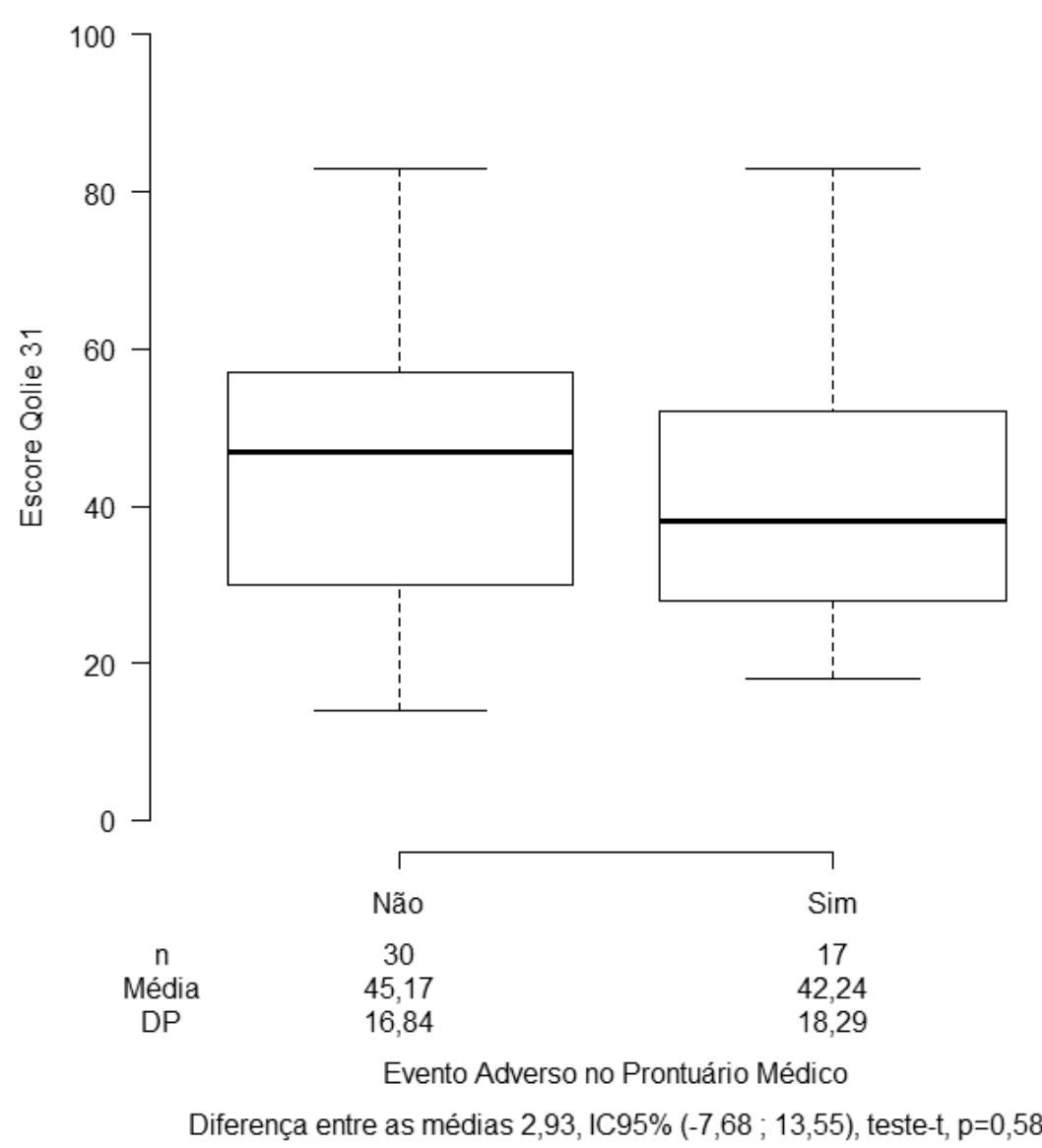

Figura 5. Associação entre a qualidade de vida (Qolie-31) e a presença de eventos adversos no prontuário médico de pacientes adultos com epilepsia refratária em uso de lamotrigina atendidos no AEDC do HCFMRP-USP no período de maio/2011 a abril/2012.

Essa divergência de resultados entre a associação de qualidade de vida e as duas formas utilizadas para análise dos eventos adversos pode ser atribuída ao subdiagnóstico durante a consulta médica e/ou à supervalorização dos pacientes quando se utiliza uma busca ativa dos eventos adversos que comprometem a qualidade de vida ${ }^{25}$.

Além destas duas questões relevantes na interpretação dos resultados, é importante destacar que estudos longitudinais que conseguiram avaliar o uso da LTG em monoterapia e comparar os efeitos humanísticos com os FAE de primeira geração evidenciaram que a LTG apresentou meIhores resultados na melhoria da qualidade de vida quando comparada com a CBZ, PHT e VPA ${ }^{24}$. Viteri e colaboradores (2010) também comprovaram, por meio de um estudo de coorte prospectivo, que a LTG apresenta impacto positivo sobre a qualidade de vida dos pacientes com epilepsia, princi- palmente no grupo das mulheres ${ }^{26}$. Frente a esta constatação, não se deve associar os baixos escores do Qolie-31 observados no presente estudo especificamente com o uso da LTG. Por fim, é válido destacar a potencialidade do estudo no que se refere a avaliação dos parâmetros em pacientes do mundo real, atendidos em clínica do Sistema Único de Saúde (SUS), no contextos da póscomercialização do medicamento. Isso torna-se uma fortaleza do trabalho, visto que a maioria dos dados existentes na literatura são oriundos de ambientes controlados e muitas vezes na fase de pré-comercialização dos medicamentos.

\section{CONCLUSÕES}

Em relação ao perfil de eventos adversos, foi observado que $44 \%$ dos pacientes apresentaram escores AEP elevados, igual ou superior a 
45 pontos, sendo os eventos adversos mais prevalentes: sonolência, dificuldade de concentração e problemas de memória;

Não se observou diferença significativa entre a média dos escores AEP do grupo que apresentou registro de evento adverso no prontuário e o grupo que não apresentou registro de eventos no prontuário médico $(p=0,24)$, sugerindo que os eventos adversos relacionados aos FAE são identificados e registrados pelos clínicos durante a consulta médica;

Os pacientes com epilepsia farmacorresistente apresentaram baixos escores em todos os domínios relacionados à qualidade de vida (Qolie31), e este parâmetro humanístico apresentou associação inversamente proporcional com os eventos adversos obtidos pelo AEP $(r=-0,69$, $p<0,01)$, sugerindo que, quanto maiores os escores do AEP, pior a qualidade de vida desses pacientes.

\section{REFERÊNCIAS}

1. Fisher RS, Acevedo C, Arzimanoglou A, Bogacz A, Cross $\mathrm{H}$, Elger $\mathrm{CE}$, et al. A practical clinical definition of epilepsy. Epilepsia. 2014; 55: 475-82.

2. Sankaraneni R, Lachhwani D. Antiepleptic drugs - a review. Pediatr Ann. 2015; 44: e36-42.

3. Ben-Menachem E. Medical management of refractory epilepsy-Practical treatment with novel antiepileptic drugs. Epilepsia. 2014; 55: 3-8.

4. González FJL, Osorio XR, Rein AGN, Martínez MC, Fernández JS, Haba VV, et al. Drug-resistant epilepsy: definition and treatment alternatives. Neurología. 2015; 30: 439-46.

5. Beyenburg S, Stavem K, Schmidt D. Placebo-corrected efficacy of modern antiepileptic drugs for refractory epilepsy: systematic review and meta-analysis. Epilepsia. 2010; 51: 7-26.

6. Perucca E, Kwan P. Overtreatment in epilepsy: adverse consequences and mechanisms. Epilepsy Res. 2002; 52: 25-33.

7. Gilliam F. Optimizing health outcomes in active epilepsy. Neurology. 2002; 58: 9-20.

8. Martins $\mathrm{HH}$, Alonso NB, Vidal-Dourado M, Carbonel TD, Araújo Filho GM, Caboclo LO, et al. Are adverse effects of antiepileptic drugs different in symptomatic partial and idiopathic generalized epilepsies? The Portuguese-Brazilian validation of the Liverpool Adverse Events Prole. Epilepsy Behav. 2011;22:511-17.

9. Da Silva TI, Ciconelli RM, Alonso NB, Azevedo AM, Westphal-Guitti AC, Pascalicchio TF, et al. Validity and reliability of the Portuguese version of the quality of life in epilepsy inventory (QOLIE-31) for Brazil. Epilepsy Behav. 2007; 10: 234-41.

10. Gilliam FG, Fessler AJ, Baker G, Vahle V, Carter J, Attarian H. Systematic screening allows reduction of adverse antiepileptic drug effects: a randomized trial. Neurology. 2004; 62: 23-7.
11. Beghi E, Gatti G, Tonini C, Ben-Menachem E, Chadwick $\mathrm{D}$, Nikaronova $\mathrm{M}$, et al. Adjunctive therapy versus alternative monotherapy in patients with partial epilepsy failing on a single drug: a multicentre, randomized, pragmatic, controlled trial. Epilepsy Res. 2003; 57: 1-13.

12. Betting LE, Kobayashi E, Montenegro MA, Min LL, Cendes F, Guerreiro MM, et al. Tratamento da epilepsia. Consenso dos especialistas brasileiros. Arq Neuropsiquiatr. 2003; 61: 1045-70.

13. Brodie MJ, Sills GJ. Combining antiepileptic drugs rational polytherapy? Seizure - European Journal of Epilepsy. 2011; 20: 369-75.

14. Stephen LJ, Brodie MJ. Antiepileptic drug monotherapy versus polytherapy: pursuing seizure freedom and tolerability in adults. Curr Opin Neurol. 2012; 25: 16472.

15. Landmark CJ, Rytter E, Johannessen SI. Clinical use of antiepileptic drugs at a referral centre for epilepsy. Seizure - European Journal of Epilepsy. 2007; 16: 356-64.

16. Malerba A, Ciampa C, Fazio S, Fattore C, Frassine B, La Neve $A$, et al. Patterns of prescription of antiepileptic drugs in patients with refractory epilepsy at tertiary referral centres in Italy. Epilepsy Res. 2010; 91: 273-82.

17. Food and drugs administration - FDA. Clobazam. [Internet] Disponível em <http:// www. accessdata.fda.gov/drugsatfda docs/label/2013/ 202067s001lbl.pdf>, acesso em 17 de julho de 2013.

18. Allen JW, Oxley J, Robertson MM, Trimble MR, Richens A, Jawad SS. Clobazam as adjunctive treatment in refractory epilepsy. Br Med J, Clin Res. Ed. 1983; 286: 1246- 7 .

19. Scheinfeld $\mathbf{N}$. Impact of phenytoin therapy on the skin and skin disease. Expert Opin Drug Saf. 2004; 3: 655-65.

20. French JA, Kanner AM, Bautista J, Abou-Khalil B, Browne $\mathrm{T}$, Harden $\mathrm{CL}$, et al. Eficacy and tolerability of the new antiepileptic drugs. II. Treatment of refractory epilepsy: report of the TTA and QSS Subcommittees of the American Academy of Neurology and the American Epilepsy Society. Epilepsia. 2004; 45: 410-23.

21. Andrew T, Milinis K, Baker G, Wieshmann U. Self reported adverse effects of mono and polytherapy for epilepsy. Seizure. 2012; 21: 610-13.

22. Wong ICK, Mawer GE, Sander JWAS. Adverse event monitoring in lamotrigine patients: a pharmacoepidemiologic study in the United Kingdom. Epilepsia. 2001; 42: 237-44.

23. Aldenkamp AP, Baker G. A Systematic Review of the Effects of Lamotrigine on Cognitive Function and Quality of Life. Epilepsy Behav. 2001; 2: 85-91.

24. Kaminow L, Schimschhock JR, Hammer AE, Vuong $A$. Lamotrigine monotherapy compared with carbamazepine, phenytoin, or valproate monotheraphy in patients with epilepsy. Epilepsy Behav. 2003;4: 659-66

25. Alexandre Junior $V$, Monteiro EA, Freitas-Lima P, Pinto KD, Velasco TR, Terra VC, et al. Addressing overtreatment in patients with refractory epilepsy at a tertiary referral centre in Brazil. Epileptic Disorder 2011; 13: 56-60.

26. Viteri C, Codina M, Cobaleda S, Lahuerta J, Barriga J. Morales MD. Quality of life and treatment satisfaction in Spanish epilepsy patients on monotherapy with lamotrigine or valproic acid. Seizure. 2010; 19: 432-8. 